\title{
Die Sicherungsverwahrung ist ein Thema für die Psychiatrie - Kontra
}

\author{
Preventive Detention is a Psychiatric Issue - Contra
}

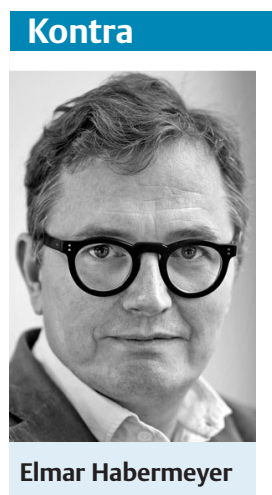

In den letzten 20 Jahren hat sich die Sicherungsverwahrung (SV) von einem juristischen Auslaufmodell zu der Maßregel entwickelt, die im Zentrum einer Vielzahl politischer Aktivitäten und juristischer Diskussionen stand. Parallel dazu ist in Deutschland die Anzahl der Sicherungsverwahrten von 176 im Jahr 1996 auf 492 Untergebrachte im Jahr 2013 gestiegen. Die Debatte darüber, was Sicherungsverwahrung ist und wie bzw. ab wann sie zu begründen sei, hat für die Psychiatrie an Bedeutung gewonnen. Insofern ist diese Maßregel natürlich ein Thema für die Psychiatrie. Offen ist jedoch, ob das medizinische Fach der Psychiatrie/Psychotherapie in der Lage ist, die ihr vom Gesetzgeber auferlegte Rolle auszufüllen:

Seit dem Urteil des Bundesverfassungsgerichts vom 4.5.2011 steht die SV vor einer grundlegenden Reform, die unmittelbare therapeutische Implikationen hat. Bis dato war für ihre Anordnung das Sicherheitsinteresse der Allgemeinheit, die vor rückfallgefährdeten, hochgefährlichen Hangtätern geschützt werden soll, entscheidend. Nun ist in Reaktion auf ein Urteil des Europäischen Gerichtshofs für Menschenrechte aus 2010, das die nachträgliche Aufhebung der bis zum Gesetz zur Bekämpfung von Sexualstraftaten gültigen Höchstfrist von 10 Jahren als nicht akzeptabel angesehen hat, in großer Eile eine Intensivierung therapeutischer Ansätze geplant bzw. umgesetzt worden. Das Bundesverfassungsgericht forderte nämlich, ohne dass dem Urteil entnommen werden kann, dass man sich mit der therapeutischen Umsetzbarkeit dieser Forderung auseinandergesetzt hätte, ein freiheitsorientiertes Gesamtkonzept der Sicherungsverwahrung mit klarer thera- peutischer Ausrichtung auf das Ziel, die von dem Untergebrachten ausgehende Gefahr zu minimieren und auf diese Weise die Dauer der Freiheitsentziehung auf das unbedingt erforderliche Maß zu reduzieren. Interessanterweise verlief auch die anschließende politische Ausgestaltung dieses Gesamtkonzepts ohne Mitwirkung von psychiatrischen und/oder psychotherapeutischen Experten, obwohl die DGPPN bereits zu einem frühen Zeitpunkt angeboten hatte, sich an den erforderlichen Überlegungen zu beteiligen [1].

Somit war die Sicherungsverwahrung im politischen Entscheidungsprozess kein Thema für die Psychiatrie. Die fehlende Mitwirkung von Therapieexperten hat den juristischen und politischen Entscheidungsträgern die sachliche und auch realistische Auseinandersetzung mit dem Adressatenkreis der von ihnen angedachten therapeutischen Bemühungen erspart. So konnte in der Diskussion außen vor bleiben, dass es sich bei den Sicherungsverwahrten um in der Regel über 50-jährige Wiederholungs- bzw. Intensivtäter mit Sexual- und/oder Gewaltdelikten handelt. Bei den Insassen wurden ein früher Beginn kriminellen Handelns, eine lange Lebenshaftzeit und zahlreiche Vorstrafen vor der Anlasstat festgestellt [2, 3]. Insgesamt zeigt sich eine Klientel, die durch ungünstige Milieueinflüsse geprägt wurde und frühzeitig, letztlich aber auch hartnäckig eine mangelhafte soziale Integration und robuste delinquente Verhaltensstile gezeigt hat. Zwar ist davon auszugehen, dass beim weitaus überwiegenden Teil der Sicherungsverwahrten eine psychiatrische Diagnose gestellt werden kann [2, 3]. Dabei handelt es sich aber nahezu regelhaft um eine Antisoziale Persönlichkeitsstörung und bislang existieren keine hinsichtlich ihrer Wirksamkeit empirisch belegten therapeutischen Konzepte $[4,5]$ zur Behandlung dieser Störung.

Wenn ein Einbezug von Fachexperten wirklich ein Thema gewesen wäre, hätten diese außerdem mitteilen können, dass die hohe Ausprägung des Merkmals psychopathy [6] bei den Insassen, deren Werte (z. B. 24 bei Basedikis et al. [7]) deutlich über den Werten deutschsprachiger Häftlingsgruppen liegen, für eine eingeschränkte therapeutische Erreichbarkeit spricht und darüber hinaus auch therapieschädigendes Verhalten erwarten lässt [8]. Ganz unabhängig davon sind angesichts des fortgeschrittenen Lebensalters und der langen Haftzeiten der Sicherungsverwahrten auch Einflüsse der Institutionalisierung zu beachten, die sich negativ auf die Behandlungsmotivation und Behandelbarkeit auswirken können.

Vor diesem Hintergrund wird das im Urteil des Bundesverfassungsgerichts angedachte Ideal in der Praxis kaum umsetzbar sein. Vielmehr besteht die Gefahr, dass die therapeutischen Bemühungen ins Leere laufen, was Fachleute ohne Weiteres hätten mitteilen können, wenn denn eine Berücksichtigung des psychiatrischpsychotherapeutischen Wissensstandes ein Thema gewesen wäre. Stattdessen blieb der von Kröber [9] skizzierte Mythos eines therapeutischen Wunderstabs, mit dem Persönlichkeitsstörungen, sexuelle Deviationen, Dissozialität, Begierden und Verhaltensstörungen geheilt werden können, intakt. Die Vermutung liegt nahe, dass das so gewollt war, denn bei Konfrontation mit den Realitäten dieser Maßregel wäre deutlich geworden, dass die Ausführungen des Bundesverfassungsgerichts allenfalls als Aufforderung angesehen werden können, Behandlungsprogramme für antisoziale Straftäter zu entwickeln und diese parallel zu ihrer Entwicklung in der Praxis zu evaluieren. Stattdessen wurde jedoch von medizinischen Laien bei nach dem gegenwärtigen Wissensstand schwer zu therapierenden Personen eine Hoffnung auf Besserung bzw. Entlassung geschürt, die vor dem Hintergrund des aktuellen medizinischpsychotherapeutischen Sachstands nicht zu begründen ist.

Wenn es wirklich um Entlassung gehen würde, wären weniger psychiatrisch-psy- 
chotherapeutische Interventionen und Therapien wie Tataufarbeitung oder Empathietraining erforderlich, sondern vielmehr ein intensives Übergangsmanagement [9] bzw. ein therapeutisch begleiteter und gut vorbereiteter bzw. engmaschig überwachter sozialer Empfangsraum [10]. Dem Gesetzgeber mag mit der Psychiatrisierung der Sicherungsverwahrung die rasche Schließung einer Sicherheitslücke nach dem Urteil des Europäischen Gerichtshofs für Menschenrechte gelungen sein. Für die unmittelbar Betroffenen, also die zukünftigen „Patienten“ und ihre „Therapeuten“, dürfte der Weg letztlich aber in eine Sackgasse führen. Aus Sicht des Autors wurde die Chance auf eine grundlegende Reform des Maßregelvollzugs in Deutschland vertan. Es steht zu befürchten, dass die Sicherungsverwahrung daher auch weiterhin ein allerdings problematisches - Thema für die Psychiatrie bleiben wird.

\section{Literatur}

1 DGPPN. Zur Entscheidung des Bundesverfassungsgerichts zu den Regelungen zur Sicherungsverwahrung vom 4.5.2011. Stellungnahme der Deutschen Gesellschaft für Psychiatrie, Psychotherapie und Nervenheilkunde (DGPPN) von Müller J, Nedopil N, Saimeh N, Schneider F, Falkai P. 2011: Online publiziert unter: http://www.dgppn.de/pub likationen/stellungnahmen/detailansicht/ select/stellungnahmen-2011/article/141/ zur-neuregel.html

2 Habermeyer E, Passow D, Puhlmann P et al. Die Maßregel der Sicherungsverwahrung: Empirische Befunde zu den Insassen und der psychiatrischen Gutachtenpraxis. Fortschr Neurol Psychiatr 2008; 76: 672 677

3 Gairing S, de Tribolet-Hardy F, Vohs K et al. Sicherungsverwahrte ( $\$ 66$ StGB) Merkmale der Täter und ihre Bedeutung für die Erfolgsaussichten eines therapeutischen Vollzugs. Nervenarzt 2013; 84: 65 - 71

4 Gibbon S, Duggan C, Stoffers J et al. Psychological interventions for antisocial personality disorder. Cochrane Database Syst Rev 2010; 6: CD007668

5 Khalifa N, Duggan C, Stoffers J et al. Pharmacological interventions for antisocial personality disorder. Cochrane Database Syst Rev 2010; 8: CD007667

Sie haben eine eigene Meinung zu diesem

Thema? Dann schreiben Sie uns an:

psychiat-praxis@thieme.de!
6 Hare RD. The Hare Psychopathy Checklist Revised (PCL-R). 2nd Ed. Toronto, ON: 2003

7 Basdekis-Jozsa R, Mokros A, Vohs K et al. Preventive detention in Germany: An overview and empirical data from two federal states. Behavioral Sciences \& the Law 2013; 31: 344-358

8 Mokros A, Habermeyer E. Behandlung von Patienten mit „psychopathy“. In: Rossegger A, Endrass J, Borchard B. Forensisch Psychologisches Risk Management. Berlin: Medizinisch wissenschaftliche Verlagsgesellschaft; 2012

9 Kröber HL. Therapie gegen Gewalt. Forensische Psychiatrie Psychologie Kriminologie 2011; 5: 64-65

10 Voß T, Sauter J, Kröber HL. Entlassene Problemfälle in der ambulanten Nachsorge von langzeitinhaftierten und langzeituntergebrachten Patienten. Forensische Psychiatrie Psychologie Kriminologie 2011; 5: 253-260

Korrespondenzadresse

\section{Prof Dr. med. Elmar Habermeyer}

Klinik für Forensische Psychiatrie

Psychiatrische Universitätsklinik Zürich

Lenggstraße 31

8032 Zürich, Schweiz

elmar.habermeyer@puk.zh.ch

Bibliografie

Dol http://dx.doi.org/

10.1055/s-0034-1387323

Psychiat Prax 2014; 41: 353-354

(c) Georg Thieme Verlag KG

Stuttgart · New York

ISSN 0303-4259 